ITEP-PH-4/2004

FZJ-IKP-TH-2004-18

\title{
Evolution of high-mass diffraction from the light quark valence component of the pomeron
}

\author{
N.N. Nikolaev ${ }^{a, b}$, W. Schäfer ${ }^{a}$, B.G. Zakharov ${ }^{b}$ and V.R. Zoller ${ }^{c}$ \\ ${ }^{a} I K P$ (Theorie), FZ Jülich, Jülich, Germany \\ ${ }^{b}$ L.D. Landau Institute for Theoretical Physics, Moscow 117940, Russia \\ ${ }^{c}$ ITEP, Moscow 117218, Russia.
}

\begin{abstract}
We analyze the contribution from excitation of the $(q \bar{q})(f \bar{f}),(q \bar{q}) g_{1} \ldots g_{n}(f \bar{f})$ Fock states of the photon to high mass diffraction in DIS. We show that the large $Q^{2}$ behavior of this contribution can be described by the DLLA evolution from the non-perturbative $f \bar{f}$ valence state of the pomeron. Although of higher order in pQCD, the new contribution to high-mass diffraction is comparable to that from the excitation of the $q \bar{q} g$ Fock state of the photon.
\end{abstract}


In this communication we report a direct evaluation of high-mass diffractive deep inelastic scattering (DIS) from excitation of the $(q \bar{q})(f \bar{f})$ Fock states of the photon, fig. 1, where $q$ and $f$ are the light quarks. The interest in this problem can be formulated as follows.

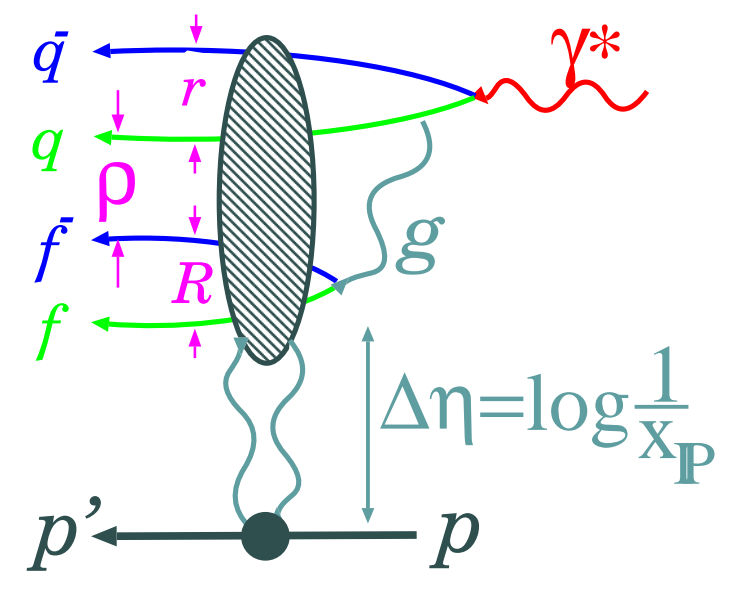

Figure 1: The color dipole structure of diffractive excitation of perturbative $(q \bar{q})(f \bar{f})$ state of the photon with the rapidity gap $\Delta \eta$ from the proton. The contribution of other diagrams with radiation of the gluon and/or splitting $g \rightarrow f \bar{f}$ after the interaction with the target vanishes to DLLA.

On the one hand, within perturbative QCD (pQCD) diffractive DIS, $\gamma^{*} p \rightarrow X+p^{\prime}$, can be described as quasi-elastic scattering and excitation of the multiparton Fock states $X$ of the incident photon of virtuality $Q^{2}[1,2]$. As such it is a manifestly nonlinear - quadratic - functional of the dipole cross section for the multiparton states, $X=q \bar{q}, q \bar{q} g, \ldots$. For the forward case, $t=0$, where $t$ is the $\left(p, p^{\prime}\right)$ momentum transfer squared,

$$
\left.\frac{d \sigma^{D}}{d t}\right|_{t=0}=\frac{1}{16 \pi}\left[\left\langle q \bar{q}\left|\sigma^{2}\right| q \bar{q}\right\rangle+\left\langle q \bar{q} g\left|\sigma_{3}^{2}-\sigma^{2}\right| q \bar{q} g\right\rangle+\ldots\right]
$$

where $\sigma$ and $\sigma_{3}$ stand for the dipole cross section for the Fock states $|q \bar{q}\rangle,|q \bar{q} g\rangle$, respectively, interacting with the proton target, evaluated at the starting point of the small- $x$ evolution, $x_{\mathbf{I P}}=x_{0}$. On the other hand, motivated by the triple-reggeon approach to diffraction excitation [3], one would like to reinterpret high-mass diffractive DIS as an inclusive DIS off the pomeron,

$$
\left.\left(Q^{2}+M^{2}\right) \frac{d \sigma^{D}}{d t d M^{2}}\right|_{t=0}=\left\langle q \bar{q}\left|\sigma^{\mathbf{P}}\left(x_{\mathbf{I P}}, \beta, \mathbf{r}\right)\right| q \bar{q}\right\rangle
$$


If possible at all, such a color dipole representation will only be meaningful if the effect of higher Fock states of the photon can consistently be reabsorbed into the small- $\beta$ evolution of $\sigma^{\mathbb{P}}\left(x_{\mathbb{P}}, \beta, \mathbf{r}\right)[2,4]$. Here $\beta=Q^{2} /\left(M^{2}+Q^{2}\right)$, where $M$ is the mass of the diffractive system, is the Bjorken variable for DIS off the pomeron, $x_{\mathbb{P}}=x / \beta$ is the rapidity gap variable - the fraction of the proton's lightcone momentum carried by the exchanged pomeron, $x=Q^{2} / 2 m \nu$ is the standard Bjorken variable for DIS off the proton and $\nu$ is the photon energy.

A priori it is not clear that the nonlinear (1) can be cast in the linear form (2). Furthermore, the expectation values of the square of the dipole cross section in (2) tend to be dominated by the contribution from large, non-perturbative, dipoles: $r \sim r_{f}=1 / m_{f} \sim 1 \mathrm{fm}$ for the excitation of $q \bar{q}$ states, which is the Born term for $\beta \sim 1$, and $\rho \sim R_{c} \sim 0.25 \mathrm{fm}$ for the Born term of high-mass, i.e., small- $\beta$, diffractive DIS: excitation of the $q \bar{q} g$ Fock states (for the determination of the propagation radius of perturbative gluons, $R_{c}$, from lattice QCD and elsewhere see $[5,6])$. Still, despite the manifestly nonperturbative Born term, the resummation of double-leading-log approximation (DLLA) - strongly ordered energy and dipole size contributions from $q \bar{q} g_{1} \ldots g_{n}$ excitation is possible and has been shown to correspond to the familiar DLLA evolution of the diffractive structure function [2]. Starting from [7], in the phenomenological studies of diffractive DIS it has become customary to apply the DGLAP evolution $[8]$ to the whole diffractive structure function $(\mathrm{SF})$

$$
f^{D(4)}\left(t=0, x_{\mathbf{P}}, \beta, Q^{2}\right)=x_{\mathbf{P}} F_{2}^{D(4)}\left(t=0, x_{\mathbf{P}}, \beta, Q^{2}\right)=\left.\frac{Q^{2}}{4 \pi^{2} \alpha_{e m}}\left(Q^{2}+M^{2}\right) \frac{d \sigma^{D}}{d t d M^{2}}\right|_{t=0}
$$

(being differential in $t$, the so-defined diffractive $\mathrm{SF}$ is dimensionfull, and $\sigma^{\mathbb{P}}\left(x_{\mathbb{P}}, \beta, \mathbf{r}\right)$ has a dimension $[\mathrm{mb}]^{2}$, but that does not affect its evolution properties. The $t$-integrated diffractive $\mathrm{SF}$ is dimensionless, but the modulation of the SF by the $\beta$-dependent diffraction slope [9] can spoil the evolution properties, and herebelow we focus on forward diffraction, $t=0$.) Although it has been argued to be plausible [10], and the DGLAP evolution analyses met certain phenomenological success $([11,12,13]$ and references therein, for the review see [14]), a direct demonstration of such a DGLAP evolution property of diffractive DIS is still missing.

The principal problem with extension of the analysis [2] to the contribution of the $(q \bar{q})(f \bar{f})$, $(q \bar{q}) g_{1} \ldots g_{n}(f \bar{f})$ Fock states is that the $f \bar{f}$ dipoles have a large non-perturbative size, $R \sim r_{f}=$ $1 / m_{f} \gg R_{c}$. The gross features of $\beta$-distribution in $\gamma^{*} p \rightarrow(f \bar{f}) p^{\prime}$ are well understood: in 
close analogy to the valence structure function of the proton, it is peaked at $\beta \sim 1 / 2$, so that only finite masses, $M^{2} \sim Q^{2}$, are excited $[1,15]$. However, it is not obvious that this non-perturbative valence $\beta$-distribution defined by $\gamma^{*} p \rightarrow(f \bar{f}) p^{\prime}$ will enter the evolution of the $q \bar{q}$ sea of the pomeron in precisely the same way as the valence quark density enters the evolution of the sea of nucleons. Here we report a direct demonstration that such a pQCD evolution holds at least to the DLLA accuracy. Furthermore, we show that although the $(q \bar{q})(f \bar{f})$ contribution is of higher order in the pQCD coupling $\alpha_{S}$, see Fig. 1, it is enhanced by a potentially large numerical factor, $\propto\left[\sigma\left(r_{f}\right) / \sigma\left(R_{c}\right)\right]^{2}$, and numerically it is comparable to the leading order $q \bar{q} g$ contribution. We report also a derivation of the inclusive spectrum of gluon jets from diffraction excitation of the $q \bar{q} g$ states of the photon, which clarifies the small-r ${ }^{2}$ scaling properties of $\sigma^{\mathbb{P}}\left(x_{\mathbf{I P}}, \beta, \mathbf{r}\right)$.

The further presentation is organized as follows. We start with the brief introduction into the color dipole description of small- $\beta$ diffraction and demonstration of the representation (2) for excitation of the $q \bar{q} g$ state. Then we show how the DLLA contribution from $(q \bar{q})(f \bar{f})$ excitation to $f^{D(4)}\left(t=0, x_{\mathbf{I P}}, \beta, Q^{2}\right)$ can be cast in the form $(2)$ with $\sigma^{\mathbb{P}}\left(x_{\mathbf{I P}}, \beta, \mathbf{r}\right)$ evaluated for scattering of the $q \bar{q}$ dipole on the $f \bar{f}$ valence state of the pomeron. We present the DLLA evaluation of the small- $\beta$ diffractive $\mathrm{SF}$, compare our results with experimental data $[12,13]$ and conclude with a brief summary.

In the color dipole QCD approach to DIS [16, 2, 4] the two principal quantities are the dipole cross section, $\sigma(x, \mathbf{r})$, for interaction of the $q \bar{q}$ dipole $\mathbf{r}$ with the proton target and the $q \bar{q}$ dipole size distribution in the projectile photon $e_{q}^{2}\left|\Psi_{\gamma^{*}}\left(Q^{2}, z, \mathbf{r}\right)\right|^{2}$. In terms of $\sigma(x, \mathbf{r})$ the cross section of inclusive DIS has the form of an expectation value over the $q \bar{q}$ Fock state, $\sigma_{\gamma^{*} p}\left(x, Q^{2}\right)=\langle q \bar{q}|\sigma(x, \mathbf{r})| q \bar{q}\rangle$, the effect of higher order perturbative Fock states, $q \bar{q} g_{1} \ldots g_{n}$, can be reabsorbed into the leading $\log (1 / x)$ color dipole BFKL evolution of $\sigma(x, \mathbf{r})$. The relationship between the dipole cross section and the unintegrated gluon structure function $\mathcal{F}\left(x, \kappa^{2}\right)=\partial G\left(x, \kappa^{2}\right) / \partial \log \kappa^{2}$, reads

$$
\sigma(x, \mathbf{r})=\frac{4 \pi \alpha_{S}\left(r^{2}\right)}{N_{c}} \int \frac{d^{2} \boldsymbol{\kappa}}{\left(\kappa^{2}+\mu_{G}^{2}\right)^{2}}[1-\exp (i \boldsymbol{\kappa} \mathbf{r})] \mathcal{F}\left(x, \kappa^{2}\right) \approx \frac{\pi^{2} \alpha_{S}\left(r^{2}\right)}{N_{c}} r^{2} G\left(x, \frac{A}{r^{2}}\right),
$$

where $R_{c}=1 / \mu_{G}$ is the Yukawa correlation radius for perturbative gluons and in the DLLA for small dipoles $A \simeq 10$. Because of $\log Q^{2}$ scaling violations $G\left(x, A / r^{2}\right)$ rises with the hard 
scale $A / \mathbf{r}^{2}$. To the lowest order in pQCD $[16,17]$

$$
\mathcal{F}\left(x, \boldsymbol{\kappa}^{2}\right)=\frac{C_{F} \alpha_{S}\left(\boldsymbol{\kappa}^{2}\right)}{\pi} \cdot N_{c} V_{N}(\boldsymbol{\kappa}),
$$

where $N_{c} V_{N}(\boldsymbol{\kappa})$ can be regarded as the number of valence partons in the proton resolved by gluons at the scale $\boldsymbol{\kappa}^{2}$. Here the vertex function $V_{N}(\boldsymbol{\kappa})=1-G_{2}(\boldsymbol{\kappa},-\boldsymbol{\kappa})$ and the two-quark form-factor of the target nucleon, $G_{2}(\boldsymbol{\kappa},-\boldsymbol{\kappa})$, vanishes for $\boldsymbol{\kappa}^{2} \gtrsim R_{N}^{-2}$, where $R_{N}$ is the radius of the nucleon.

The $q \bar{q} g$ contribution to (1) describes the first iteration of the $\log (1 / \beta)$ evolution of diffractive DIS and can be separated into the radiative correction to the small-mass $q \bar{q}$ excitation and the Born term of the high-mass $q \bar{q} g$ excitation as follows. Let $\mathbf{r}, \boldsymbol{\rho}$ and $\boldsymbol{\rho}-\mathbf{r}$ be the $\bar{q}-q, g-q$ and $g-\bar{q}$ separations in the impact parameter (transverse size) plane. The $q \bar{q} g$ 3-body interaction cross section equals [2]

$$
\sigma_{3}(x, \mathbf{r}, \boldsymbol{\rho})=\frac{C_{A}}{2 C_{F}}[\sigma(x, \boldsymbol{\rho})+\sigma(x, \boldsymbol{\rho}-\mathbf{r})]-\frac{1}{N_{c}^{2}-1} \sigma(x, \mathbf{r}) .
$$

For soft perturbative gluons carrying a small fraction of photon's momentum, $z_{g} \ll 1$, and Yukawa infrared regularization the wave function of the 3-parton $q \bar{q} g$ state equals $[2,4]$

$$
\begin{aligned}
& \left|\Phi\left(\mathbf{r}, \boldsymbol{\rho}, z, z_{g}\right)\right|^{2}= \\
& \frac{e_{q}^{2} C_{F} \alpha_{S}\left(r^{2}\right)}{\pi^{2} z_{g}}\left|\Psi_{\gamma^{*}}\left(Q^{2}, z, \mathbf{r}\right)\right|^{2}\left|\mu_{G} K_{1}\left(\mu_{G} \rho\right) \frac{\boldsymbol{\rho}}{\rho}-\mu_{G} K_{1}\left(\mu_{G}|\boldsymbol{\rho}-\mathbf{r}|\right) \frac{\boldsymbol{\rho}-\mathbf{r}}{|\boldsymbol{\rho}-\mathbf{r}|}\right|^{2} \\
& =\frac{e_{q}^{2}}{z_{g}} \cdot\left|\Psi_{\gamma^{*}}\left(Q^{2}, z, \mathbf{r}\right)\right|^{2} \cdot \mathcal{K}(\boldsymbol{\rho}-\mathbf{r}, \boldsymbol{\rho}) \simeq \frac{e_{q}^{2} C_{F} \alpha_{S}\left(r^{2}\right)}{\pi^{2} z_{g}}\left|\Psi_{\gamma^{*}}\left(Q^{2}, z, \mathbf{r}\right)\right|^{2} \frac{r^{2}}{\rho^{4}} F\left(\mu_{G} \rho\right)
\end{aligned}
$$

where $\mathcal{K}(\boldsymbol{\rho}-\mathbf{r}, \boldsymbol{\rho})$ is the kernel of the color dipole BFKL equation

$$
\begin{aligned}
\frac{\partial \sigma(x, \mathbf{r})}{\partial \log \frac{1}{x}}=\mathcal{K} \otimes \sigma(x, \mathbf{r}) & =\frac{2 C_{F}}{C_{A}} \int d^{2} \boldsymbol{\rho} \mathcal{K}(\boldsymbol{\rho}-\mathbf{r}, \boldsymbol{\rho})\left[\sigma_{3}(x, \mathbf{r}, \boldsymbol{\rho})-\sigma(x, \mathbf{r})\right] \\
& =\int d^{2} \boldsymbol{\rho} \mathcal{K}(\boldsymbol{\rho}-\mathbf{r}, \boldsymbol{\rho})[\sigma(x, \boldsymbol{\rho})+\sigma(x, \boldsymbol{\rho}-\mathbf{r})-\sigma(x, \mathbf{r})]
\end{aligned}
$$

and we also showed the DLLA approximation for $\mathbf{r}^{2} \ll \boldsymbol{\rho}^{2}$. The form factor of the infrared cutoff, $F(z)$, satisfies $F(0)=1$ and $F(z) \propto \exp (-2 z)$ at $z>1[2,7]$.

Now notice, that in view of (7) the $q \bar{g} g$ contribution to(1) can be rearranged as

$$
\mathcal{K} \cdot\left(\sigma_{3}^{2}-\sigma^{2}\right)=2 \sigma \mathcal{K} \cdot\left(\sigma_{3}-\sigma\right)+\mathcal{K} \cdot\left(\sigma_{3}-\sigma\right)^{2}
$$


The first term in the rhs of (9) is the radiative correction to the small-mass $q \bar{q}$ excitation with the rapidity gap $x_{\mathbf{I P}} \sim x$, i.e. the contribution from the 2-parton state to the total cross section of diffraction (1) must be calculated with the BFKL-evolved

$$
\sigma\left(x_{\mathbb{I}}, \mathbf{r}\right)=\sigma\left(x_{0}, \mathbf{r}\right)+\int_{x_{\mathbb{P}}}^{x_{0}} \frac{d x^{\prime}}{x^{\prime}} \mathcal{K} \otimes \sigma\left(x^{\prime}, \mathbf{r}\right)
$$

The second term in (9) is the Born tern of the high-mass 3-parton, $q \bar{q} g$, excitation with the rapidity gap $x_{\mathbb{P}}=x_{0}$. In the high-mass regime $d z_{g} / z_{g}=d M^{2} /\left(M^{2}+Q^{2}\right)$ and after undoing the $z_{g}$-integration the DLLA 3-parton cross section takes the form

$$
\begin{aligned}
& \left.\left(Q^{2}+M^{2}\right) \frac{d \sigma_{q \bar{q} g}^{D}}{d t d M^{2}}\right|_{t=0}= \\
& \frac{1}{16 \pi} \int d z d^{2} \mathbf{r} d^{2} \boldsymbol{\rho}\left\{z_{g}\left|\Phi\left(\mathbf{r}, \boldsymbol{\rho}, z, z_{g}\right)\right|^{2}\right\}_{z_{g}=0} \cdot\left[\sigma_{3}\left(x_{\mathbf{I P}}, \mathbf{r}, \boldsymbol{\rho}\right)-\sigma\left(x_{\mathbf{I P}}, \mathbf{r}\right)\right]^{2} \\
\simeq \quad & \frac{1}{16 \pi} \int d z d^{2} \mathbf{r}\left|\Psi_{\gamma^{*}}\left(Q^{2}, z, \mathbf{r}\right)\right|^{2} \frac{\pi^{2} \alpha_{S}\left(r^{2}\right)}{N_{c}} r^{2} \cdot \frac{C_{A}^{2} N_{c}}{C_{F}} \int_{r^{2}} d^{2} \boldsymbol{\rho}\left[\frac{\sigma\left(x_{\mathbf{P}}, \boldsymbol{\rho}\right)}{\rho^{2}}\right]^{2} F\left(\mu_{G} \rho\right) .
\end{aligned}
$$

It gives a flat small- $\beta$ behaviour of $f^{D(4)}\left(t=0, x_{\mathbf{I P}}, \beta, Q^{2}\right)$ with the strength controlled $[2,15]$ by non-perturbative large $\rho$, cut off from above at $\rho \sim R_{c}$ by the nonperturbative form factor $F\left(\mu_{G} \rho\right)$. The small- $\rho$ integration can safely be extended to $\rho=0$, so that $(11)$ is of the desired color dipole form (2) and can be treated as DIS off the sea generated by perturbative splitting of gluons from the valence $g g$ state of the pomeron. There is one caveat, though: the gluon density in the pomeron defined by eq. (11),

$$
G_{g g}^{\mathbf{P}}\left(x_{\mathbf{P}}, \beta\right)=\frac{C_{A}^{2} N_{c}}{C_{F}} \cdot \int d^{2} \boldsymbol{\rho}\left[\frac{\sigma\left(x_{\mathbf{P}}, \boldsymbol{\rho}\right)}{\rho^{2}}\right]^{2} F\left(\mu_{G} \rho\right),
$$

is short of the collinear scaling violations present in (4).

The extension of the above analysis to the DLLA description of diffractive excitation of the high-mass $(q \bar{q})(f \bar{f})$ Fock state of the photon proceeds as follows. As we shall see a posteriori, the DLLA contribution comes from $\mathbf{r}^{2} \ll \rho^{2} \ll \mathbf{R}^{2}$. First we recall that the $q \bar{q}$ excitation is dominated by very asymmetric pairs, in which one of the final partons carries a very small fraction of the photons momentum, $z \sim m_{q}^{2} / Q^{2} \ll 1$, so that in the impact parameter space the fast parton with $\bar{z}=1-z$ flies along the photon's trajectory, while the slower parton is at large transverse distance $r \sim 1 / m_{q}$ from the parent photon [1]. Consequently, the fast parton of the $f \bar{f}$ shall have the same impact parameter as the gluon radiated by the parent $q \bar{q}$ dipole. 
In view of the DLLA ordering, $\mathbf{r}^{2} \ll \boldsymbol{\rho}^{2} \ll \mathbf{R}^{2}$, the partons of the parent $q \bar{q}$ dipole and the fast parton of the radiative $f \bar{f}$ pair can be treated as the pointlike (anti)triplet color charge, and the $(q \bar{q})(f \bar{f})$ state interacts with the target nucleon as the $f \bar{f}$ dipole with the dipole cross section $\sigma\left(x_{\mathbf{I P}}, \mathbf{R}\right)$. The distribution of $f \bar{f}$ color dipoles in the gluon of transverse momentum $\boldsymbol{\kappa}$ is identical to that in the photon subject to the substitutions $N_{c} \alpha_{e m} e_{f}^{2} \rightarrow T_{F} \alpha_{S}\left(\boldsymbol{\kappa}^{2}\right)$ and $Q^{2} \rightarrow \boldsymbol{\kappa}^{2}$, so that the diffractive cross section of interest equals

$$
\left.\left(Q^{2}+M^{2}\right) \frac{d \sigma_{(q \bar{q})(f \bar{f})}^{D}}{d t d M^{2}}\right|_{t=0}=\frac{1}{16 \pi} \int d^{2} \boldsymbol{\kappa} \frac{d g_{q \bar{q}}\left(Q^{2}, \boldsymbol{\kappa}\right)}{d^{2} \boldsymbol{\kappa}} \cdot \frac{T_{F} \alpha_{S}\left(\boldsymbol{\kappa}^{2}\right)}{N_{c} \alpha_{e m} e_{f}^{2}}\left\langle f \bar{f}\left|\sigma^{2}\left(x_{\mathbf{I P}}, \mathbf{R}\right)\right| f \bar{f}\right\rangle
$$

where the flux of gluons in the parent $q \bar{q}$ state is given by the momentum-space version of (7):

$$
\frac{d g_{q \bar{q}}\left(Q^{2}, \boldsymbol{\kappa}\right)}{d^{2} \boldsymbol{\kappa}}=\int_{0}^{1} d z_{q} \int d^{2} \mathbf{r}\left|\Psi_{\gamma^{*}}\left(Q^{2}, z_{q}, \mathbf{r}\right)\right|^{2} \frac{2 e_{q}^{2} C_{F} \alpha_{S}\left(r^{2}\right)}{\pi^{2}} \cdot \frac{[1-\exp (i \boldsymbol{\kappa} \mathbf{r})]}{\left(\boldsymbol{\kappa}^{2}+\mu_{G}^{2}\right)^{2}} \boldsymbol{\kappa}^{2}
$$

Finally, notice that

$$
\begin{aligned}
\frac{\boldsymbol{\kappa}^{2}}{4 \pi^{2} \alpha_{e m}} \cdot \frac{1}{16 \pi e_{f}^{2}}\left\langle f \bar{f}\left|\sigma^{2}\left(x_{\mathbf{I P}}, \mathbf{R}\right)\right| f \bar{f}\right\rangle & =\left.\frac{\boldsymbol{\kappa}^{2}}{4 \pi^{2} \alpha_{e m}} \cdot \frac{d \sigma\left(\gamma^{*}\left(\boldsymbol{\kappa}^{2}\right) \rightarrow f \bar{f}\right)}{d t}\right|_{t=0} \\
=\frac{1}{e_{f}^{2}} \int_{0}^{1} \frac{d \beta}{\beta} f_{f \bar{f}}^{D(4)}\left(t=0, x_{\mathbf{I P}}, \beta, \boldsymbol{\kappa}^{2}\right) & =N_{f \bar{f}}^{\mathbf{P}_{\mathbf{P}}}\left(x_{\mathbf{I P}}, \boldsymbol{\kappa}^{2}\right)
\end{aligned}
$$

where $N_{f \bar{f}}^{\mathbb{P}_{\bar{f}}}\left(x_{\mathbf{P}}, \boldsymbol{\kappa}^{2}\right)$ can be reinterpreted as a number of charged valence partons, i.e., twice the number of $f \bar{f}$ dipoles, in the pomeron. Upon the substitution of (15) and (14) into (13) one readily recovers the dipole representation (2), in which $\sigma^{\mathbb{P}}\left(x_{\mathbf{P}}, \beta, \mathbf{r}\right)$ is evaluated from equation (4) in which unintegrated gluon density (5) is substituted for by the unintegrated gluon density evolved from the $f \bar{f}$ state of the pomeron

$$
\mathcal{F}_{f \bar{f}}^{\mathbb{P}_{f}}\left(\beta, \boldsymbol{\kappa}^{2}\right)=\frac{C_{F} \alpha_{S}\left(\boldsymbol{\kappa}^{2}\right)}{\pi} N_{f \bar{f}}^{\mathbf{P}_{(}}\left(x_{\mathbf{I P}}, \boldsymbol{\kappa}^{2}\right)
$$

Furthermore, $N_{f \bar{f}}^{\mathbf{P}_{(}}\left(x_{\mathbf{I P}}, \boldsymbol{\kappa}^{2}\right)$ vanishes at $\boldsymbol{\kappa}^{2}=0$ and, according to $[16,1]$, flattens at $\boldsymbol{\kappa}^{2} \gg$ $m_{f}^{2}$ which, in comparison to (5) suggests the transverse size of the $f \bar{f}$ component of the pomeron $r_{f} \sim 1 / m_{f}$. One can come to the same conclusion from the point that the dominant contribution to (14) comes from $f \bar{f}$ dipoles with $R \sim 1 / m_{f}$.

The DLLA analysis of $q \bar{q} g_{1} \ldots g_{n}$ excitation developed in [2] can readily be extended to the higher, $(q \bar{q}) g_{1} . . g_{n}(f \bar{f})$, states. The crucial point is that to DLLA the $f \bar{f}$ dipole is the largest 
one, so that the corresponding contribution to the diffractive cross section is still given by equation (13) where the DLLA evolution is reabsorbed into the flux of gluon $g_{n}$, which is the softest with respect to the photon. Viewed from the pomeron side, that amounts to the DLLA small- $\beta$ evolution of $\sigma^{\mathbb{P}}\left(x_{\mathbb{P}}, \beta, \mathbf{r}\right)$ with the boundary condition defined by gluon density (16). As such, the emerging $\log ^{n-1}(1 / \beta) \cdot \log ^{n}\left(1 / \alpha_{S}\left(r^{2}\right)\right)$ structure of DLLA expansion in the energy and collinear logarithms for diffractive SF from $(q \bar{q}) g_{1} . . g_{n}(f \bar{f})$ excitation is identical to DLLA structure of the proton SF. As shown in [2], DLLA expansion for diffractive SF from $(q \bar{q}) g_{1} . . g_{n}$ excitation is of a marginally different structure $\log ^{n-1}(1 / \beta) \cdot \log ^{n-1}\left(1 / \alpha_{S}\left(r^{2}\right)\right)$. Besides that, the two components of the diffractive structure function have a manifestly different $x_{\mathbf{I P}}$-dependence [7]: driven by $\sigma\left(x_{\mathbb{I P}}, r_{f}\right)$ in $(16)$ for the $q \bar{q},(q \bar{q})(f \bar{f}),(q \bar{q}) g_{1} . . g_{n}(f \bar{f})$ excitations and by $\sigma\left(x_{\mathbf{I P}}, R_{c}\right)$ for the $(q \bar{q}) g_{1} . . g_{n}$ excitations. This concludes the proof of the DLLA small- $\beta$ evolution at fixed $x_{\mathbf{I P}}$ of such a two-component diffractive structure function $f^{D(4)}\left(t=0, x_{\mathbf{I P}}, \beta, Q^{2}\right)$.

The absence of a scaling violation in (11) and (12) implies that in contrast to (5) the corresponding unintegrated gluon density $\mathcal{F}_{g g}^{D}\left(\beta, \boldsymbol{\kappa}^{2}\right)$ vanishes for large $\boldsymbol{\kappa}^{2}$. A closely related observable is the transverse momentum, p, spectrum of semihard diffractive gluons with $\mathbf{p}^{2} \ll \mathbf{r}^{-2} \ll Q^{2}[18]$. Since $\mathbf{p}$ is a variable conjugate to $\boldsymbol{\rho}$, upon the relevant Fourier transforms

$$
\begin{aligned}
\left(Q^{2}+M^{2}\right) & \left.\frac{d \sigma^{D}\left(\gamma^{*} \rightarrow g X\right)}{d t d M^{2} d^{2} \mathbf{p}}\right|_{t=0}=\frac{1}{16 \pi(2 \pi)^{2}} \int d z d^{2} \mathbf{r} C_{F}\left|\Psi_{\gamma^{*}}(z, \mathbf{r})\right|^{2} \frac{\alpha_{S}(r)}{\pi^{2}} \\
& \times\left|\int d^{2} \boldsymbol{\rho} \exp (i \mathbf{p} \boldsymbol{\rho})\left[\frac{\boldsymbol{\rho}}{\boldsymbol{\rho}^{2}}-\frac{\boldsymbol{\rho}-\mathbf{r}}{(\boldsymbol{\rho}-\mathbf{r})^{\mathbf{2}}}\right]\left[\sigma_{3}\left(x_{\mathbf{P}}, r, \boldsymbol{\rho}\right)-\sigma\left(x_{\mathbf{P}}, \mathbf{r}\right)\right]\right|^{2} \\
= & \int d z d^{2} \mathbf{r}\left|\Psi_{\gamma^{*}}(z, \mathbf{r})\right|^{2} \frac{\pi \alpha_{S}\left(r^{2}\right)}{N_{c}} r^{2} \cdot \frac{C_{A}^{2}}{4 C_{F} N_{c}} \cdot\left[\frac{\alpha_{S}\left(\mathbf{p}^{2}\right) G\left(x_{\mathbf{P}}, \mathbf{p}^{2}\right)}{\mathbf{p}^{2}}\right]^{2}
\end{aligned}
$$

Within the reinterpretation of diffraction as DIS of pomerons, the $\mathbf{p}$ has a meaning of intrinsic transverse momentum in the valence $g g$ state of the pomeron. Indeed, the spectrum (17) falls steeper than the $1 / \mathbf{p}^{2}$ spectrum of gluons from inclusive DIS off a nucleon.

The numerical results for high-mass, small- $\beta$, diffraction depend on the input dipole cross section $\sigma(x, \mathbf{r})$. Here we evaluate the lowest order $q \bar{q} g$ and $(q \bar{q})(f \bar{f})$ contributions to diffractive DIS in a specific color dipole BFKL model [4, 19] which gives a good description of the proton SF data. The applicability domain of the small- $\beta$, small- $x_{\mathbf{I P}}$ formalism is $\beta, x_{\mathbf{I P}}<$ 


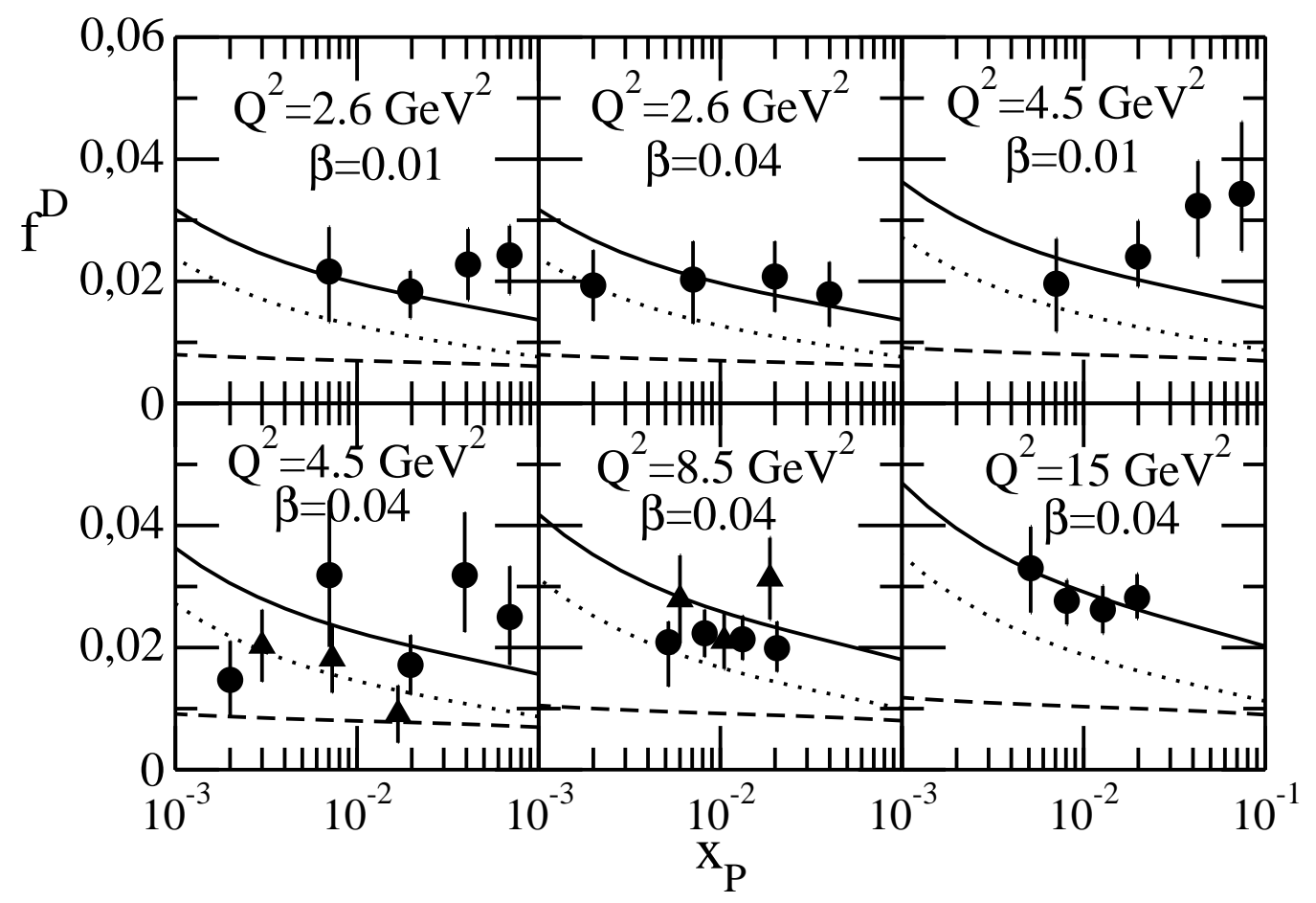

Figure 2: The comparison with the experimental data on small- $\beta$, small- $x_{\mathbf{I P}}$ diffractive structure function ([12], full circles; [13], full triangle) of the theoretical evaluation of $f^{D(3)}=f_{q \bar{q} g}^{D(3)}+f_{(q \bar{q})(f \bar{f})}^{D(3)}$ shown by the solid line. The dotted line corresponds to $f_{q \bar{q} g}^{D(3)}$ and the dashed line represents $f_{(q \bar{q})(f \bar{f})}^{D(3)}$.

$x_{0} \ll 1$, the experience with inclusive DIS suggests $x_{0} \sim 0.03$, although the theoretical curves in fig. 2 are stretched up to $x_{\mathbf{I P}}=0.1$. This small- $\beta$, small- $x_{\mathbf{I P}}$ domain is almost at the boundary of the HERA experiments, the corresponding experimental data on the $t$ integrated diffractive structure function $f^{D(3)}\left(x_{\mathbf{I P}}, \beta, Q^{2}\right)$ from $\mathrm{H} 1$ ([12], circles) and ZEUS ([13], triangles) are shown in fig. 2. We evaluate this structure function as $f^{D(3)}\left(x_{\mathbf{I P}}, \beta, Q^{2}\right)=$ $\int d t f^{D(4)}\left(t, x_{\mathbf{P}}, \beta, Q^{2}\right) \approx \frac{1}{B_{3 \mathbb{P}}} f^{D(4)}\left(t=0, x_{\mathbf{I P}}, \beta, Q^{2}\right)$ with the central value of the diffraction slope $B_{\mathbf{I P}}=B_{D}=7.2 \pm 1.1_{-0.9}^{+0.7} \mathrm{GeV}^{-2}$ as reported by ZEUS [20]. The apparent growth of the experimentally observed $f^{D(3)}\left(x_{\mathbf{I P}}, \beta, Q^{2}\right)$ towards large $x_{\mathbf{I P}} \sim 0.1$ is usually attributed to the non-vacuum admixture to the pomeron exchange. Two features of the theoretical results 
for small- $\beta$ diffraction are noteworthy. First, the contributions from $q \bar{q} g$ and higher-order $(q \bar{q})(f \bar{f})$ states are of comparable magnitude because $R_{c} \ll r_{f}$ and the latter is enhanced $\propto\left[\sigma\left(x_{\mathbb{P}}, r_{f}\right) / \sigma\left(x_{\mathbb{P}}, R_{c}\right)\right]^{2}$. Second, because of the same inequality of the important dipole sizes, $R_{c} \ll r_{f}$, the $x_{\mathbf{P}}$-dependence of the $q \bar{q} g$ excitation is steeper than that of the $(q \bar{q})(f \bar{f})$ excitation. This point has been made already in [7], the numerically significant contribution

from the $(q \bar{q})(f \bar{f})$ excitation makes the overall $x_{\mathbb{P}^{-}}$-dependence of $f^{D(3)}\left(x_{\mathbb{P}}, \beta, Q^{2}\right)$ weaker than evaluated in [7] for the pure $q \bar{q} g$ excitation. The solid curve in fig. 2 is the combined contribution from the two mechanisms. It is in reasonably good agreement with the HERA data.

To summarize, we reported the first explicit proof of the DLLA evolution property of the contribution to diffractive structure function from excitation of $(q \bar{q})(f \bar{f}),(q \bar{q}) g_{1} \ldots g_{n}(f \bar{f})$ Fock states of the photon. We demonstrated that the corresponding diffractive SF can be cast in the color dipole representation. The boundary condition for the DLLA small- $\beta$ evolution is provided by the Born dipole cross section built perturbatively upon the valence $f \bar{f}$ state of the pomeron, as defined by the $\gamma^{*} p \rightarrow(f \bar{f}) p^{\prime}$ excitation, in precisely the same manner as in inclusive DIS off the nucleon starting with the valence quark distribution. Compared to the $q \bar{q} g$ excitation, the $(q \bar{q})(f \bar{f})$ is of higher order to pQCD. Still the numerical evaluations confirm the expectation that the pQCD $\alpha_{S}$ suppression is compensated for by the larger dipoles in the $(q \bar{q})(f \bar{f})$ state compared to the $q \bar{q} g$ state of the photon.

This work has been partly supported by the INTAS grant 00-00366 and the DFG grant 436RUS17/72/03.

\section{References}

[1] N.N. Nikolaev and B.G. Zakharov, Z. Phys. C53 (1992) 331.

[2] N.N. Nikolaev and B.G. Zakharov, J. Exp. Theor. Phys. 78 (1994) 598 [Zh. Eksp. Teor. Fiz. 105 (1994) 1117]. Z.Phys. C64 (1994) 631.

[3] K.A.Ter-Martirosyan, Phys. Lett. B44 (1973) 179;

A.B.Kaidalov and K.A.Ter-Martirosyan, Nucl. Phys. B75 (1974) 471; G. Ingelman and 
P. E. Schlein, Phys. Lett. B 152 (1985) 256.

[4] N.N.Nikolaev, B.G.Zakharov and V.R.Zoller, JETP Letters 59 (1994) 8; Phys. Lett. B328 (1994) 486; Zh. Exp. Teor. Fiz. 105 (1994) 1498; N.N. Nikolaev and B.G. Zakharov, Phys. Lett. B327 (1994) 149; B327 (1994) 157.

[5] E. Meggiolaro, Phys. Lett. B 451 (1999) 414.

[6] J. H. Field, Phys. Rev. D 66 (2002) 013013.

[7] M. Genovese, N.N. Nikolaev, B.G. Zakharov J. Exp. Theor. Phys. 81 (1995) 625 [Zh. Eksp. Teor. Fiz. 108 (1995) 1141]; Phys. Lett. B 378 (1996) 347; Phys. Lett. B 380 (1996) 213.

[8] V. N. Gribov and L. N. Lipatov, Yad. Fiz. 15 (1972) 781 [Sov. J. Nucl. Phys. 15 (1972) 438]; Y. L. Dokshitzer, Sov. Phys. JETP 46 (1977) 641 [Zh. Eksp. Teor. Fiz. 73 (1977) 1216]; G. Altarelli and G. Parisi, Nucl. Phys. B 126, 298 (1977).

[9] N. N. Nikolaev, A. V. Pronyaev and B. G. Zakharov, JETP Lett. 68 (1998) 634.

[10] A. Berera and D. E. Soper, Phys. Rev. D 53 (1996) 6162; J. C. Collins, Phys. Rev. D 57 (1998) 3051 [Erratum-ibid. D 61 (2000) 019902]; M. Grazzini, L. Trentadue and G. Veneziano, Nucl. Phys. B 519 (1998) 394; L. Trentadue and G. Veneziano, Phys. Lett. B 323 (1994) 201.

[11] C. Royon, L. Schoeffel, J. Bartels, H. Jung and R. Peschanski, Phys. Rev. D 63 (2001) 074004 .

[12] H1 Collab., Measurements of the Diffractive Structure Function $F_{2}^{D(3)}$ at HERA, submitted to XX Int. Symp. on Lepton and Photon Interactions, July 23, 2001, Rome; Z. Phys. C76 (1997) 613.

[13] ZEUS Collab., J. Breitweg et al., Eur. Phys. J. C6 (1999) 43.

[14] A. Hebecker, Phys. Rept. 331 (2000) 1. 
[15] M. Genovese, N.N. Nikolaev, B.G. Zakharov Phys. Lett. B378 (1996) 347; N.N. Nikolaev, A.V.Pronyaev, B.G. Zakharov Phys.Rev. D59 (1999) 091501.

[16] N.N. Nikolaev and B.G. Zakharov, Z. Phys. C49 (1991) 607.

[17] N. N. Nikolaev and B. G. Zakharov, Phys. Lett. B 332 (1994) 177.

[18] N. N. Nikolaev, Hamburg 1998/1999: Monte Carlo generators for HERA physics, pp. 377-381. e-Print Archive: hep-ph/9905562

[19] N.N.Nikolaev, B.G.Zakharov and V.R.Zoller, JETP Lett. 66 (1997) 138; N.N.Nikolaev, J. Speth and V.R.Zoller, Phys. Lett. B473 (2000) 157; J. Exp. Theor. Phys. 93 (2001) 957; V.R. Zoller, Phys. Lett. B509 (2001) 69.

[20] ZEUS Collab., J. Breitweg et al., Eur. Phys. J. C1 (1998) 81. 